Invent. math. 123, $611(1996)$

Inventiones

mathematicae

(C) Springer-Verlag 1996

\title{
Erratum
}

\section{Zero-cycles on quadric fibrations: Finiteness theorems and the cycle map}

\author{
R. Parimala, V. Suresh
}

Invent. math. 122, 83-117 (1995)

The above article unfortunately contained the following error which was introduced when it was processed for printing.

On pp. $83,86,89,90,92,93,95,97,98,101,115$, the sign $\leqq$ was changed to an italic number 5 .

The publisher deeply regrets this mistake.

Springer-Verlag 